\title{
Asociación del estado nutricional y factores clínicos con muerte relacionada con tuberculosis en Colombia
}

\author{
Association of nutritional status and clinical factors with tuberculosis related death \\ in Colombia
}

\author{
Soraya Salas Romero', Jaime Lorduy Gómez² y Ana Belen Simancas Salemi
}

'Programa de Medicina, Facultad de Ciencias de la Salud, Corporación Universitaria Rafael Núñez, Cartagena, Colombia.
2Programa de Instrumentación Quirúrgica, Facultad de Ciencias de la Salud, Corporación Universitaria Rafael Núñez, ARGOS QUIRÚRGICO Cartagena, Colombia.
Estudiante Programa de Medicina, semillerista GINUMED, Facultad de Ciencias de la Salud, Corporación Universitaria Rafael Núñez, Cartagena, Colombia.

Financiamiento: Corporación Universitaria Rafael Núñez.

Los autores declaran no tener conflictos de interés para este artículo.

Recibido: 27 de julio de 2020 / Aceptado: 8 de marzo de 2021

\section{Resumen}

Introducción: Estudios relacionan la tuberculosis (TBC) con características socio-demográficas de las poblaciones humanas y la presencia de co-morbilidades en estos pacientes. Hoy, una de las co-morbilidades más importantes en Colombia es la desnutrición; sería importante verificar su relación con el tema de mortalidad, independientemente de las demás variables presentes en los pacientes. Objetivo: Determinar la asociación del estado nutricional y los principales factores clínicos con la muerte relacionada a TBC en Colombia. Pacientes y Métodos: Se realizó un estudio descriptivo de corte transversal sobre un total de 12.778 pacientes con acotados criterios de inclusión y exclusión. Se realizó análisis bivariado y se construyó modelo multivariado de regresión logística. Resultados: Las variables socio-demográficas asociadas con muerte por TBC fueron: sexo masculino (OR 1,49 IC 95\% 1,14-1,95); población indígena (OR 1,76 IC 95\% 1,09-2,85). Las variables clínicas: bajo peso (OR 1,32 IC $95 \%$ 1,04-1,68) y co-infección por VIH (OR 1,46 IC 95\% $1,06-2,00)$ se asociaron a mayor mortalidad; como factor protector detectamos tener formas de TBC extra-pulmonar (OR 0,44 IC 95\% 0,29-0,67) Conclusiones: En pacientes con bajo peso, co-infección por VIH, sexo masculino y población indígena se presentó un alto riesgo de muerte por TBC, por lo que se hace necesario implementar estrategias de abordaje integral.

Palabras clave: tuberculosis; estado nutricional; mortalidad; factores de riesgo.

\begin{abstract}
Background: Studies link tuberculosis (TB) with sociodemographic characteristics of human populations and the presence of comorbidities in these patients. Today one of the most important comorbidities in Colombia is malnutrition; it would be important to verify its relationship with the mortality issue independently of the other variables present in the patients. Aim: To determine the association of nutritional status and the main clinical factors with death related to TB in Colombia. Methods: A descriptive cross-sectional study was carried out in a total of 12,778 patients who met defined inclusion and exclusion criteria. Bivariate analysis was performed and a multivariate logistic regression model was constructed. Results: The sociodemographic variables associated with death from TB were: male sex (OR 1.49 95\% CI 1.14-1.95); indigenous population (OR $1.7695 \%$ CI 1.09-2.85). The clinical variables: low weight (OR 1.32 95\% CI 1.04-1.68) and clothing for HIV (OR 1.46 95\% CI 1.06-2.00) were related to mortality and having forms of extrapulmonary TB were as a protective factor (OR $0.4495 \%$ CI 0.29-0.67). Conclusions: In patients with low weight, HIV coinfection, male sex, and indigenous population presented a high risk of death from TB, making it necessary to implement comprehensive approach strategies.
\end{abstract}

Keywords: tuberculosis; nutritional status; mortality; risk factors. 


\section{Introducción}

L a tuberculosis (TBC) continúa siendo una de las diez principales causas de muerte en el mundo y la principal causa de un solo agente infeccioso por encima del VIH; sin embargo, la carga de la enfermedad a nivel mundial está disminuyendo ${ }^{1}$. La proporción de personas con TBC que murieron a causa de ésta en el año 2017 fue de $16 \%$, frente al $23 \%$ que se registró en el 2000 , evidenciando una lenta disminución en las cifras de muertes, que podría no ser suficiente para alcanzar las metas propuestas en la estrategia "Fin a la TBC" ",

Esta estrategia incluye el manejo integral del paciente con TBC y enuncia la importancia de conocer el estatus nutricional para establecer un adecuado control del mismo $^{2}$, puesto que la asociación entre TBC y la malnutrición puede determinar el resultado de la infección. La malnutrición no sólo está asociada a la desnutrición y la hipernutrición, sino también a afecciones derivadas de desequilibrios dietéticos que son causa de enfermedades no transmisibles relacionadas con la dieta.

Cabe anotar que la desnutrición aumenta la susceptibilidad a la enfermedad; así mismo, la infección aumenta la demanda de energía metabólica como respuesta corporal a la infección y cambios en el metabolismo de los macronutrientes. En otras palabras, esto lleva a producir un círculo vicioso sinérgico del estado nutricional adverso. En este mismo sentido existen reportes en los que se relaciona un mayor riesgo de muerte entre los pacientes con TBC y bajo peso ${ }^{3,4}$, por lo que debe considerarse un manejo nutricional del paciente complementario al tratamiento farmacológico contra la $\mathrm{TBC}^{5-7}$.

La TBC es la infección oportunista más común en personas infectadas por VIH y la causa de muerte de una de cada tres personas con SIDA en el mundo ${ }^{8}$. La infección por VIH aumenta el riesgo de TBC y, a su vez, la TBC acelera la morbi-mortalidad asociada al $\mathrm{VIH}^{9}$. Esta susceptibilidad se ve incrementada por la desnutrición, la que provoca deterioro del sistema inmune por el incremento del gasto energético, la malabsorción y la deficiencia de micronutrientes, incrementando la susceptibilidad, la progresión de la enfermedad y el riesgo de muerte ${ }^{10}$.

Diferentes estudios relacionan la mortalidad por TBC con características socio-demográficas de los pacientes (edad, sexo, pertenencia étnica, entre otras) y la presencia de co-morbilidades ${ }^{11-14}$. Sería importante saber si en Colombia, independientemente de las variables que tradicionalmente se asocian con muerte, se puede establecer al mismo tiempo una asociación con los casos de desnutrición, a fin de facilitar la formulación de estrategias ajustadas que permitan la consecución de las metas del programa.

Es objetivo del presente estudio determinar la aso- ciación del estado nutricional y los principales factores clínicos con la muerte relacionada a TBC en Colombia.

\section{Pacientes y Métodos}

Se realizó un estudio descriptivo de corte transversal; respecto a la temporalidad del estudio se realizó un análisis retrospectivo con fuente de información secundaria. La información se recolectó de las bases de datos notificados en el año 2016 de los eventos TBC y TBC farmacoresistente del Sistema de Vigilancia de Salud Pública de Colombia (SIVIGILA), los cuales fueron suministrados por el Instituto Nacional de Salud (INS) mediante acuerdo de transferencia de información. Un total de 13.887 casos de TBC y TBC farmacoresistente fueron notificados en 2016, de los cuales 12.778 cumplían los criterios de selección.

Entre los criterios de inclusión se encontraron tener más de 18 años y ser notificado como un caso confirmado de uno de los siguientes eventos: TBC pulmonar, extrapulmonar o farmacoresistente. Los criterios de exclusión consideraron a mujeres gestantes y aquellos pacientes que no registraran información en la variable índice de masa corporal (IMC), peso y/o talla. La clasificación del estado nutricional de los pacientes se estableció de acuerdo al valor del índice de masa corporal (IMC) documentado en la ficha de notificación, categorizándolos en bajo peso (IMC < 18,5), normopeso (IMC 18,5 a 25), sobrepeso (IMC > 25), acorde a los parámetros establecidos por la Organización Mundial de la Salud ${ }^{15}$.

La mortalidad fue categorizada en dos grupos: muerte específicamente asociada a TBC (que incluyó a los pacientes que registraron como causa básica de defunción los siguientes códigos CIE 10 [A150-A199; B909; M011]) $\mathrm{y}$ otras causas de muerte.

Las variables consideradas en el estudio se clasificaron en tres macrovariables principales: socio-demográficas, aspectos clínicos de los pacientes y formas de TBC -comorbilidades. Para el análisis univariado se calcularon la frecuencia y porcentajes para las variables cualitativas y se presentaron en tablas resúmenes de frecuencia. Para el análisis bivariado se utilizó la prueba de asociación estadística $\chi^{2}$ con corrección de Pearson; para el cruce de variables cualitativas politómicas o de las dicotómicas con valores esperados mayores de cinco en cada celda. Se manejó esta misma prueba con corrección de Fisher, para las variables dicotómicas, con al menos un valor esperado menor de cinco. Se determinó por el análisis multivariado, la fuerza de asociación calculando los valores de la razón de disparidad y sus respectivos intervalos de confianza, utilizando métodos de regresión logística binaria. El análisis de la información se realizó mediante el software SPSS V24. 
y mujeres fue $21 \mathrm{~kg} / \mathrm{m}^{2}$ y $21,7 \mathrm{~kg} / \mathrm{m}^{2}$, respectivamente; el $27 \%$ de los pacientes se encontraban en bajo peso.

El 48,6\% de los pacientes requirió hospitalización, encontrándose que $21,5 \%$ tenía edades mayores a 65 años; de ellos, $25,4 \%$ tenía bajo peso.

El $56,9 \%$ de los casos de TBC se confirmó con una baciloscopia positiva.

Para el caso de las formas clínicas de TBC, 85,8\% eran TBC pulmonar -en ellos se observó al mayor porcentaje (29\%) de pacientes con bajo peso- y 14,1\% evidenció formas extra-pulmonares.

El grupo poblacional más importante es la población carcelaria $(5,4 \%)$, seguido de la población indígena $(4,8 \%)$; en este último se evidenció el mayor porcentaje de pacientes con bajo peso $(29,2 \%)$ (Tabla 1$)$.

\section{Resultados}

En los 12.778 pacientes con TBC del estudio se observó un promedio de edad de 47,4 años ( $\mathrm{DS} \pm 19$ ), además, que $64,4 \%$ de los pacientes eran hombres. En $2,4 \%$ de los notificados la condición final reportada fue muerte asociada a TBC. La media del IMC de los hombres

\section{Mortalidad atribuible a TBC y variables socio- demográficas}

El análisis de las variables socio-demográficas evidenció relaciones importantes con los casos de muertes asociadas a TBC Se observaron diferencias estadísticamente significativas $(p=0,0001)$ entre los promedios de

\begin{tabular}{|c|c|c|c|c|}
\hline & \multicolumn{2}{|c|}{ Media } & \multicolumn{2}{|c|}{ DS } \\
\hline Edad & \multicolumn{2}{|c|}{47,4} & \multicolumn{2}{|c|}{ $\pm 19,0$} \\
\hline IMC & \multicolumn{2}{|c|}{21,5} & \multicolumn{2}{|c|}{ $\pm 4,6$} \\
\hline Características & $\begin{array}{c}\text { Bajo peso } \\
\mathbf{f}(\%)\end{array}$ & $\begin{array}{c}\text { Normopeso } \\
\mathbf{f}(\%)\end{array}$ & $\begin{array}{c}\text { Sobrepeso } \\
f(\%)\end{array}$ & $\begin{array}{l}\text { Total } \\
\mathrm{f}(\%)\end{array}$ \\
\hline Hombres & $2.257(27,4 \%)$ & $4.851(58,9 \%)$ & $1.123(13,6 \%)$ & $8.231(64,4 \%)$ \\
\hline Mujeres & $1.205(26,5 \%)$ & $2.374(52,2 \%)$ & $968(21,3 \%)$ & $4.547(35,6 \%)$ \\
\hline Mayores de 65 años & $698(25,4 \%)$ & $1.475(53,6 \%)$ & $578(21,0 \%)$ & $2.751(21,5 \%)$ \\
\hline Baciloscopias positivas & $2.200(30,2 \%)$ & $4.115(56,5 \%)$ & $967(13,3 \%)$ & $7.282(56,9 \%)$ \\
\hline Tuberculosis pulmonar & $3.191(29,0 \%)$ & $6.116(55,8 \%)$ & $1.662(15,2 \%)$ & $10.969(85,8 \%)$ \\
\hline Tuberculosis farmacoresistente & $98(24,4 \%)$ & $252(62,8 \%)$ & $51(12,7 \%)$ & $401 \quad(3,1 \%)$ \\
\hline Diabetes mellitus & $11(12,8 \%)$ & $51(59,3 \%)$ & $24(27,9 \%)$ & $86 \quad(0,6 \%)$ \\
\hline Enfermedad renal & $1 \quad(6,7 \%)$ & $9(60 \%)$ & $5(33,3 \%)$ & $15 \quad(0,1 \%)$ \\
\hline Infección por VIH & $568(32,3 \%)$ & $1.004(57,1 \%)$ & $187(10,6 \%)$ & $1.759(13,7 \%)$ \\
\hline Enfermedad mental & $7(23,3 \%)$ & $21(70,0 \%)$ & $2(6,7 \%)$ & $30 \quad(0,2 \%)$ \\
\hline Muertes asociadas a tuberculosis & $115(37,0 \%)$ & $167(53,7 \%)$ & $29 \quad(9,3 \%)$ & $311(2,4 \%)$ \\
\hline Muertes por otras causas & $284(34,7 \%)$ & $437(53,4 \%)$ & $97(11,9 \%)$ & $818(6,4 \%)$ \\
\hline Indígena & $180(29,2 \%)$ & $372(60,3 \%)$ & $65(10,5 \%)$ & $617(4,8 \%)$ \\
\hline Discapacitados & $46(41,8 \%)$ & $48(43,6 \%)$ & $16(14,5 \%)$ & $110(0,8 \%)$ \\
\hline Población carcelaria & $177(25,3 \%)$ & $472(67,4 \%)$ & $51 \quad(7,3 \%)$ & $700 \quad(5,4 \%)$ \\
\hline Paciente hospitalizado & $1.922(30,9 \%)$ & $3.459(55,6 \%)$ & $836(13,4 \%)$ & $6.217(48,6 \%)$ \\
\hline
\end{tabular}


las edades de aquellos pacientes que murieron por causa asociada a TBC $(58,25 \mathrm{DS} \pm 19,1)$ respecto a aquellos que sobrevivieron a la infección $(47,16 \mathrm{DS} \pm 18,9)$. En los pacientes mayores de 65 años se evidenció igualmente dicha asociación $(\mathrm{p}=0,00001)$ en relación con las muertes encontradas.

Se encontró asociación estadísticamente significativa con paciente de sexo masculino $(p=0,0001)$ y aquellos que presentan alguna discapacidad $(\mathrm{p}=0,0072)$ con la posibilidad de tener muerte relacionada a TBC. No se evidencian asociaciones estadísticamente significativas, en el análisis bivariado, con la condición de vivir en cabecera municipal $(\mathrm{p}=0,416)$, en centro poblado $(\mathrm{p}=0,404)$ o en zonas rurales $(0,070)$. De igual forma, el ser desplazado $(p=0,542)$ o migrante $(0,403)$, no representan condiciones asociadas a la mortalidad por TBC (Tabla 2).

\section{Mortalidad atribuible a TBC y aspectos clínicos generales}

Es interesante destacar la asociación con el bajo peso $(\mathrm{p}=0,00007)$ y la condición de estar hospitalizados $(\mathrm{p}=0,00001)$ como factores relacionados con mortalidad atribuida a TBC.

El análisis relativo al índice de masa corporal también mostró diferencias estadísticamente significativas $(\mathrm{p}=0,0001)$ entre los promedios del IMC de los pacientes que murieron por causa asociada a TBC $(20,22 \mathrm{DS} 4,13)$ respecto a aquellos que sobrevivieron a la infección (21,56 DS 4,60).

Para el resto de factores clínicos no se observó una asociación estadísticamente significativa con los casos de muertes por TBC como es el caso de los pacientes nuevos $(p=0,116)$, es decir, aquel que recibe tratamiento

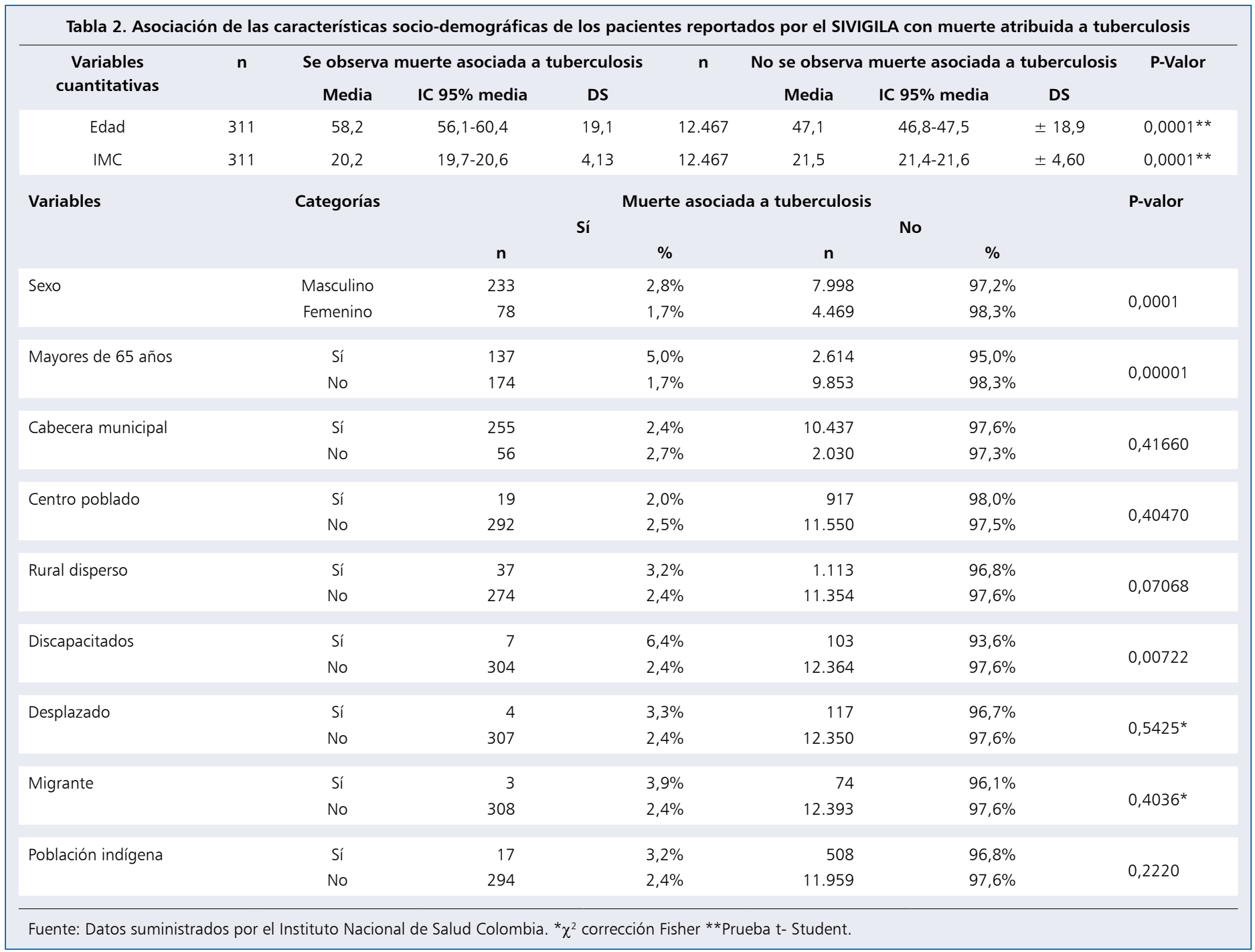


por primera vez, o haber tenido una recaída $(\mathrm{p}=0,519)$ e incluso, en aquellos en los que se inició con un nuevo tratamiento $(\mathrm{p}=0,147)$. Para la condición de paciente bacilífero $(\mathrm{p}=0,432)$ no se evidenció relación alguna con la mortalidad (Tabla 3).

\section{Mortalidad atribuida a TBC y formas clínicas de TBC y co-morbilidades}

Es importante establecer que no se evidencia asociación estadísticamente significativa con muerte, en aquellos pacientes con TBC farmacoresistente $(\mathrm{p}=$ $0,683)$ y aquellos que tenían diagnóstico de diabetes mellitus confirmada $(\mathrm{p}=0,468)$. Sin embargo, existe una asociación estadísticamente significativa en los casos de TBC extra-pulmonar $(p=0,00298)$ y en aquellos pacientes que tienen infección por VIH $(\mathrm{p}=0,027)$ con la mortalidad atribuida a TBC en la población de estudio (Tabla 4).

En cuanto a las co-morbilidades, la mayor prevalencia era la presencia de infección por VIH $(13,7 \%)$ seguido de diabetes mellitus $(0,6 \%)$.

Para esta fase del análisis se continuó con el análisis multivariante, usando la regresión logística binaria, en la que se consideraron aquellas variables que en el análisis bivariado presentaron una asociación estadísticamente significativa y aquellas que, aunque no se encontraran asociadas, tienen importancia y relevancia clínica con la presencia de muerte atribuida a TBC.

Se consideró el análisis de multi-co-linealidad, a fin de no incluir variables que no aporten al modelo. Para ello se ajustó por edad, población carcelaria, población discapacitada y pacientes hospitalizados, mostrando el riesgo de tener muerte específica por TBC en la población que tiene bajo peso (OR 1,32 IC 95\% 1,04-1,68), población del sexo masculino (OR 1,49 IC 95\% 1,14-1,95), los pacientes que tiene infección por el VIH (OR 1,46 IC 95\% $1,06-2,00)$ y los pacientes que son de población indígena (OR 1,76 IC 95\% 1,09-2,85). También se encontró como factor protector para el riesgo de muerte por TBC, tener formas de TBC extra-pulmonar (OR 0,44 IC 95\% 0,290,67 ) (Tabla 5).

\begin{tabular}{|c|c|c|c|c|c|c|}
\hline \multirow[t]{2}{*}{ Variables } & \multirow[t]{2}{*}{ Categorías } & \multicolumn{4}{|c|}{ Muerte asociada a tuberculosis } & \multirow[t]{2}{*}{ P-valor } \\
\hline & & $\mathbf{n}$ & $\%$ & $\mathbf{n}$ & $\%$ & \\
\hline \multirow[t]{2}{*}{ Bajo peso } & Sí & 115 & 3,3 & 3.347 & 96,7 & \multirow{2}{*}{0,00007} \\
\hline & No & 196 & 2,1 & 9.120 & 97,9 & \\
\hline \multirow{2}{*}{ Normopeso } & Sí & 167 & 2,3 & 7.058 & 97,7 & \multirow[b]{2}{*}{0,30555} \\
\hline & No & 144 & 2,6 & 5.409 & 97,4 & \\
\hline \multirow[t]{2}{*}{ Sobrepeso } & Sí & 29 & 1,4 & 2.062 & 98,6 & \multirow[b]{2}{*}{0,0006} \\
\hline & No & 282 & 2,6 & 10.405 & 97,4 & \\
\hline \multirow{2}{*}{$\begin{array}{l}\text { Paciente con tratamiento } \\
\text { por primera vez }\end{array}$} & Sí & 265 & 2,4 & 10.987 & 97,6 & \multirow{2}{*}{0,11680} \\
\hline & No & 46 & 3,0 & 1.480 & 97,0 & \\
\hline \multirow[t]{2}{*}{ Recaída } & Sí & 18 & 2,8 & 621 & 97,2 & \multirow[b]{2}{*}{0,51910} \\
\hline & No & 293 & 2,4 & 11.846 & 97,6 & \\
\hline \multirow[t]{2}{*}{ Otro retratamiento } & Sí & 28 & 3,2 & 859 & 96,8 & \multirow[b]{2}{*}{0,14750} \\
\hline & No & 283 & 2,4 & 11.608 & 97,6 & \\
\hline \multirow[t]{2}{*}{ Paciente hospitalizado } & Sí & 275 & 4,4 & 5.942 & 95,6 & \multirow{2}{*}{0,00001} \\
\hline & No & 36 & 0,5 & 6.525 & 99,5 & \\
\hline \multirow[t]{2}{*}{ Baciloscopias positivas } & Sí & 184 & 2,5 & 7.098 & 97,5 & \multirow[b]{2}{*}{0,43270} \\
\hline & No & 127 & 2,3 & 5.369 & 97,7 & \\
\hline
\end{tabular}

Tabla 4. Asociación de los aspectos relacionados con las formas de tuberculosis y co-morbilidades de los pacientes reportados por el SIVIGILA, en el 2016, con muerte atribuida a tuberculosis

\begin{tabular}{|c|c|c|c|c|c|c|}
\hline \multirow[t]{3}{*}{ Variables } & \multirow[t]{3}{*}{ Categorías } & \multicolumn{4}{|c|}{ Muerte asociada a tuberculosis } & \multirow[t]{3}{*}{ P-valor } \\
\hline & & & & & & \\
\hline & & $\mathbf{n}$ & $\%$ & $\mathbf{n}$ & $\%$ & \\
\hline \multirow{2}{*}{$\begin{array}{l}\text { Tuberculosis } \\
\text { farmacorresistente }\end{array}$} & Sí & 11 & 2,7 & 390 & 97,3 & \multirow[b]{2}{*}{0,68300} \\
\hline & No & 300 & 2,4 & 12.077 & 97,6 & \\
\hline \multirow[t]{2}{*}{ Diabetes mellitus } & Sí & 3 & 3,5 & 83 & 96,5 & \multirow[b]{2}{*}{$0,46816^{*}$} \\
\hline & No & 308 & 2,4 & 12.384 & 97,6 & \\
\hline \multirow[t]{2}{*}{ Infección por VIH } & Sí & 56 & 3,2 & 1.703 & 96,8 & \multirow[b]{2}{*}{0,02790} \\
\hline & No & 255 & 2,3 & 10.764 & 97,7 & \\
\hline
\end{tabular}

Tabla 5. Resultados del análisis de regresión logística del efecto de las variables independientes sobre los casos de muertes asociadas a tuberculosis ajustado por edad, sexo, paciente con VIH, tuberculosis extra-pulmonar, bajo peso, indígena, población carcelaria y discapacitada

\begin{tabular}{lcccccc}
\hline Variables & OR crudo & LI & IC 95\% & LS & $\begin{array}{c}\text { OR ajustado } \\
\text { (Exp } \boldsymbol{\beta})\end{array}$ & $\begin{array}{c}\text { IC 95\% para (EXP } \boldsymbol{\beta} \text { ) } \\
\text { LS }\end{array}$ \\
Bajo peso & & 1,599 & 1,266 & 2,019 & 1,324 & 1,040 \\
Sexo masculino & 1,669 & 1,288 & 2,163 & 1,498 & 1,685 \\
Paciente con infección por VIH & 1,388 & 1,035 & 1,862 & 1,460 & 1,955 \\
Población Indígena & 1,361 & 0,828 & 2,237 & 1,767 & 1,064 & 1,095 \\
\hline
\end{tabular}

Ajuste por las variables independientes: edad, población carcelaria, población discapacitada y pacientes hospitalizados. Fuente: Datos suministrados por el Instituto Nacional de Salud Colombia. LI: límite inferior. LS: límite superior. 


\section{Discusión}

En el presente estudio donde se incluyeron 12.778 casos de TBC confirmados que fueron notificados al Sistema de Vigilancia de Salud de Colombia, 27\% de los casos presentaban desnutrición al momento del diagnóstico de TBC definida por un IMC inferior a $18,5 \mathrm{~kg} / \mathrm{m}^{2}$. La prevalencia de malnutrición varía según publicaciones; en estudios desarrollados en La India, la prevalencia de desnutrición registra valores entre $62^{4}$ y $85 \%{ }^{16}$ de los pacientes evaluados. En pacientes hospitalizados ${ }^{17}$ y no hospitalizados ${ }^{18}$ de Brasil, la prevalencia de desnutrición fue de 36,1 y $50 \%$, respectivamente. En México, los reportes de desnutrición en este tipo de pacientes varían desde $56,8^{19}$ a $14,4 \%{ }^{11}$. La prevalencia de desnutrición en población de Taiwán de $27 \%{ }^{20}$ y de $14,4 \%$ en Nepal ${ }^{21}$ son las más similares a las encontradas en este estudio. En Colombia, un estudio reporta que la TBC fue 4,48 veces más frecuente entre los pacientes que cursan con IMC inferior a $18,5 \mathrm{~kg} / \mathrm{m}^{222}$.

Para el caso de las características socio-demográficas analizadas, se encontró una asociación estadísticamente significativa entre la muerte asociada a TBC y la edad avanzada definida como mayores de 65 años; dicha relación puede deberse a la presencia de mayor número de co-morbilidades o al retraso en el diagnóstico, ya que en esta población es frecuente que cursen con alteraciones en la presentación de los síntomas y con hallazgos radiológicos atípicos ${ }^{13,23-25}$.

Después de controlar factores de confusión, la mortalidad específica por TBC se asoció significativamente con el bajo peso al momento del diagnóstico, consistente con los hallazgos de Yen y cols. ${ }^{3,20}$. En este mismo sentido, otros autores reportan asociación entre bajo peso y muerte por todas las causas en pacientes con $\mathrm{TBC}^{4,16,26-28}$. La alta mortalidad en este grupo de pacientes puede ser debida a la disminución de la inmunidad, ya que el bajo peso puede suprimir la estimulación linfocítica y reducir la secreción de citoquinas pro-inflamatorias ${ }^{7,29,30}$.

Los resultados de nuestro estudio también revelaron una asociación entre el sexo masculino y la mortalidad específica por TBC, los que pueden explicase a la luz de recientes reportes en los que se relacionan susceptibilidad diferencial a la infección por Mycobacterium tuberculosis entre hombres y mujeres, los efectos de las hormonas esteroides sexuales sobre la respuesta inmune, las variantes genéticas y las características metabólicas ${ }^{12,31-33}$. Sin embargo, características socio-culturales como la adherencia al tratamiento, el abuso de alcohol, fumar y el uso de los sistemas de salud también podrían explicar nuestros resultados.

Respecto al mayor riesgo de mortalidad especifica por TBC entre población indígena reportado en el presente estudio, se ha relacionado con la presencia de barreras culturales asociadas a un patrón diferente de curación de la enfermedad, transmisión activa de M. tuberculosis, limitado acceso a los servicios de salud causando retraso en el diagnóstico y tratamientos incompletos ${ }^{34,35,36}$. Estudios realizados en Argentina ${ }^{14}$, Chile $^{37}$ y México ${ }^{38}$ demostraron mayor riesgo de muerte entre la población indígena en relación con poblaciones no indígenas; a pesar de que en Colombia se ha informado una alta prevalencia de TBC en la población indígena ${ }^{39}$, es recomendable desarrollar investigaciones más profundas que evidencien la situación de esta población dada la poca información disponible.

Con relación a las limitaciones de este estudio, es importante decir que, al tener un diseño descriptivo transversal y retrospectivo con información secundaria, es posible que exista sub-registro o información incompleta en algunas de las variables estudiadas; sin embargo, las conclusiones son válidas considerando que se trabajó con toda la población de pacientes de Colombia de todo un año y se utilizaron métodos de regresión logística que permitieron el ajuste de las posibles variables confundentes.

\section{Conclusiones}

En los pacientes con bajo peso, independientemente de tener co-infección con VIH, el ser de sexo masculino y de población indígena, se encontró un riesgo significativamente mayor de muerte por TBC, por lo que se requiere formular estrategias integrales en el manejo de este tipo de pacientes a fin de disminuir la mortalidad por este evento.

\section{Referencias bibliográficas}

1.- World Health Organisation. Global tuberculosis report 2018. Geneva: World Health Organization; 2018. Available from: https:// www.who.int/tb/publications/global_report/en/

2.- World Health Organisation. Implementing the end TB strategy: the essentials. Geneva: World Health Organization; 2015. Available from: https://www.who.int/tb/publications/2015/

The_Essentials_to_End_TB/en/

3.- Yen Y-F, Chuang P-H, Yen M-Y, Lin S-Y, Chuang P, Yuan M-J, et al.

Association of body mass index with tuberculosis mortality. Medicine (Baltimore) 2016; 95 (1): e2300. doi: 10.1097/ MD.0000000000002300

4.- Laux T S, Patil S. Predictors of tuberculosis treatment outcomes among a retrospective cohort in rural, Central India. J Clin Tuberc Other Mycobact Dis. 2018; 12: 41-7. doi: 10.1016/j.jctube.2018.06.005.

5.- Balinda I G, Sugrue D D, Ivers L C. More than malnutrition: a review of the relationship between food insecurity and tuberculosis. Open Forum Infect Dis. 2019; 6 (4): 1-10. doi: 10.1093/ofid/ofz102. 
6.- WHO. Guideline: Nutritional care and support for patients with tuberculosis. World Health Org. 2013; 54. Available from: http://apps.who.int/iris/ bitstream/10665/94836/1/9789241506410_eng. pdf.

7.- Schaible U E, Kaufmann S H E. Malnutrition and infection: complex mechanisms and global impacts. PLoS Med. 2007; 4 (5): e115. doi: 10.1371/journal.pmed.0040115.

8.- Tiberi S, Carvalho A C C, Sulis G, Vaghela D, Rendon A, Mello F C d. Q, et al. The cursed duet today: tuberculosis and HIV-coinfection. Press Medicale. 2017; 46 (2): e23-39. doi: 10.1016/j.lpm.2017.01.017

9.- Tornheim J A, Dooley K E. Tuberculosis associated with HIV infection. Microbiol Spectr. 2017; 5 (1): 1-16. doi: 10.1128/ microbiolspec.TNMI7-0028-2016.

10.- Sánchez J R, Díaz D, Verga B, Sánchez Luanda, Alfonso P R. Evaluación del estado nutricional en pacientes VIH/SIDA del municipio San Cristóbal. Rev Ciencias Médicas Pinar del Río. 2009; 3 (2): 113-22. Available from: http:// scielo.sld.cu/pdf/rpr/v13n2/rpr13209.pdf.

11.- Rashak H A, Sánchez-Pérez H J, Abdelbary B E, Bencomo-Alerm A, Enriquez-Ríos $\mathrm{N}$, Gómez-Velasco A, et al. Diabetes, undernutrition, migration and indigenous communities: tuberculosis in Chiapas, Mexico. Epidemiol Infect. 2019; 147: e71. doi: 10.1017/ S0950268818003461.

12.- Tan W, Soodeen-Lalloo A K, Chu Y, Xu W, Chen F, Zhang J, et al. Sex influences the association between haemostasis and the extent of lung lesions in tuberculosis. Biol Sex Differ. 2018; 9 (1): 44. doi: 10.1186/s13293-018-02039.

13.- Waitt C J, Squire S B. A systematic review of risk factors for death in adults during and after tuberculosis treatment [Review article]. Int J Tuberc Lung Dis. 2011; 15 (7): 871-85. doi: $10.5588 /$ ijtld. 10.0352 .

14.- Zerbini E, Greco A, Estrada S, Cisneros M, Colombo C, Beltrame S, et al. Risk factors associated with tuberculosis mortality in adults in six provinces of Argentina. Med (Buenos Aires). 2017; 77: 267-73. PMID: 28825568

15.- WHO. Physical status : the use of and interpretation of anthropometry, report of a WHO expert committee [Internet]. Geneva, Switzerland; 1993 [cited 2019 Sep 12]. Available from: https://apps.who.int/ iris/bitstream/handle/10665/37003/WHO TRS_854.pdf? sequence $=1$ \&isAllowed $=\mathrm{y}$.

16.- Bhargava A, Chatterjee M, Jain Y, Chatterjee B, Kataria A, Bhargava M, et al. Nutritional status of adult patients with pulmonary tuberculosis in rural central India and its association with mortality. Noymer A, editor. PLoS One. 2013; 8 (10): e77979. doi: 10.1371/journal. pone. 0077979 .
17.- Lazzari T K, Forte G C, Silva D R. Nutrition status among HIV-positive and HIV-negative inpatients with pulmonary tuberculosis. Nutr Clin Pract. 2018; 33 (6): 858-64. doi: 10.1002/ ncp. 10006 .

18.- Gomes Nunes Piva S, Nascimento Costa M da C, Rodrigues Barreto F, Martins Pereira S. Prevalence of nutritional deficiency in patients with pulmonary tuberculosis. J Bras Pneumol. 2013; 39 (4): 476-83. doi: 10.1590/S180637132013000400012 .

19.- Núñez-Rocha G M, Salinas-Martínez A M, Villarreal-Ríos E, Garza-Elizondo M E, González-Rodríguez F. Riesgo nutricional en pacientes con tuberculosis pulmonar: ¿cuestión del paciente o de los servicios de salud? Salud Publica Mex. 2000; 42 (2): 126-32. Available from: http://www.scielosp. org/scielo.php?script=sci_arttext\&pid=S0036$36342000000200008 \& \operatorname{lng}=$ es\&nrm=iso\&tlng= es.

20.- Yen Y-F, Tung F-I, Ho B-L, Lai Y-J. Underweight increases the risk of early death in tuberculosis patients. Br J Nutr. 2017; 118 (12): 1052-60. doi: 10.1017/S0007114517003166.

21.- Gurung L M, Bhatt L D, Karmacharya I, Yadav D K. Dietary practice and nutritional status of tuberculosis patients in Pokhara: a cross sectional study. Front Nutr. 2018; 5: 63. doi: 10.3389/fnut.2018.00063.

22.- Beltrán-León M, Péres-Lanos F, Sánchez L, Parra-López C, Navarrete M, Sánchez R, et al. Prevalencia y factores asociados a la tuberculosis y las micobacteriosis en pacientes positivos para HIV en Bogotá. Biomédica. 2018; 38: 120-7. doi: https://doi.org/10.7705/ biomedica.v38i0.3410.

23.- Sánchez M A, Pino J, Pacheco R, García $\mathrm{J}$ F. Análisis de letalidad en pacientes con diagnóstico de tuberculosis en un centro de alta complejidad en Cali, Colombia. Rev Chilena Infectol. 2018; 35 (2): 133-9. http://dx.doi. org/10.4067/s0716-10182018000200133

24.- Lee J, Woo Nam H, Ha Choi S, Soo Yoo S, Yup Lee $\mathrm{S}$, Ick Cha S, et al. Comparison of early and late tuberculosis deaths in Korea. J Korean Med Sci. 2017; 32: 700-3. doi: 10.3346/ jkms.2017.32.4.700

25.- Kwon Y-S, Kim Y H, Song J-U, Jeon K, Song J, Ryu Y J, et al. Risk factors for death during pulmonary tuberculosis treatment in Korea: a multicenter retrospective cohort study. J Korean Med Sci. 2014; 29 (9): 1226-31. A doi: 10.3346/jkms.2014.29.9.1226.

26.- Lai H-H, Lai Y-J, Yen Y-F. Association of body mass index with timing of death during tuberculosis treatment. Dowdy DW, editor. PLoS One. 2017; 12 (1): e0170104. Available from: https://dx.plos.org/10.1371/journal. pone.0170104.

27.- Podewils L J, Holtz T, Riekstina V, Skripconoka V, Zarovska E, Kirvelaite G, et al. Impact of malnutrition on clinical presentation, clinical course, and mortality in MDR-TB patients. Epidemiol Infect. 2011; 139 (1): 113-20. doi: 10.1017/S0950268810000907.

28.- Waitt C J, Squire S B, Yen Y-F, Chuang PP-H, Yen M-Y, Lin S-Y, et al. Risk factors for death during pulmonary tuberculosis treatment in Korea: a multicenter retrospective cohort study. J Korean Med Sci. 2009; 68 (12): 871-85. https://doi.org/10.3346/jkms.2014.29.9.1226.

29.- Cegielski J P, McMurray D N. The relationship between malnutrition and tuberculosis: evidence from studies in humans and experimental animals. Int J Tuberc Lung Dis. 2004; 8 (3): 286-98. PMID: 15139466.

30.- Chandrasekaran P, Saravanan N, Bethunaickan R, Tripathy S. Malnutrition: modulator of immune responses in tuberculosis. Front Immunol. 2017; 8: 1316. doi: 10.3389/ fimmu.2017.01316.

31.- Dibbern J, Eggers L, Schneider B E. Sex differences in the C57BL/6 model of Mycobacterium tuberculosis infection. Sci Rep. 2017; 7 (1): 10957. https://www.nature.com/ articles/s41598-017-11438-z.pdf.

32.- Bini E I, Mata Espinosa D, Marquina Castillo B, Barrios Payán J, Colucci D, Cruz A F, et al. The influence of sex steroid hormones in the immunopathology of experimental pulmonary tuberculosis. Mariño-Ramírez L, editor. PLoS One. 2014; 9 (4): e93831. https://doi. org/10.1371/journal.pone.0093831.

33.- Neyrolles O, Quintana-Murci L. Sexual inequality in tuberculosis. PLoS Med. 2009; 6 (12): e1000199. https://doi.org/10.1371/journal. pmed.1000199.

34.- Tollefson D, Bloss E, Fanning A, Redd J T, Barker K, McCray E. Burden of tuberculosis in indigenous peoples globally: a systematic review. Int J Tuberc Lung Dis. 2013; 17 (9): 1139. doi: $10.5588 / \mathrm{ijtld} .12 .0385$.

35.- Hernández Sarmiento J M, Dávila Osorio V L, Martínez Sánchez L M, Restrepo Serna L, Grajales Ospina D C, Toro Montoya AE, et al. Tuberculosis in indigenous communities of Antioquia, Colombia: epidemiology and beliefs. J Immigr Minor Health. 2013; 15 (1): 10-6. doi: 10.1007/s10903-012-9688-1.

36.- Puerto D, Erazo L, Zabaleta A, Murcia M I, Llerena C, Puerto G. Characterization of clinical isolates of Mycobacterium tuberculosis from indigenous peoples of Colombia. Biomédica. 2019; 39 (Supl. 2): 78-92. doi: 10.7705/biomedica.v39i3.4318.

37.- Pedrero M M, Oyarce A M. Una metodología innovadora para la caracterización de salud de las poblaciones indígenas de Chile: limitaciones y potencialidades. Notas Poblacion. 2009; 89. Available from: https://repositorio.cepal.org/ bitstream/handle/11362/12859/np89119145 es.pdf? sequence $=1 \&$ isAllowed $=y$.

38.- Meza-Palmeros J A, Sánchez-Perez H, 
Freyermunth-Enciso G, Sánchez-Ramírez G. El gradiente socioeconómico de la mortalidad por tuberculosis en México (2004-2008). Población y salud en Mesoamérica. 2013; 10 (2). https:// www.redalyc.org/pdf/446/44625652006.pdf.

39.- Ministerio de la Protección Social, Instituto
Nacional de Salud, Agencia Canadiense para el Desarrollo Internacional, Organización Paramericana de la Salud/Organización Mundial de la Salud. Tuberculosis en los pueblos indígenas de Colombia: el reto de la prevención y el control. Organizacion
Panamericana de la Salud. 2007. https:// www.paho.org/col/index.php?option=com docman\&view=download\&category slug=publicaciones-ops-omscolombia\&alias $=35$-tuberculosis-en-lospueblos-indigenas-de-colombia\&Itemid= 688 . 